\title{
Search for Scintillation in Doped Lead Fluoride Crystals
}

\author{
Rihua Mao, Member, IEEE, Liyuan Zhang, Member, IEEE, and Ren-Yuan Zhu, Senior Member, IEEE
}

\begin{abstract}
An effort has been made to introduce scintillation light in lead fluoride crystals by selective doping. It was found that some rare earth ions doped in the crystal may serve as luminescence centers. The photo- and $\mathrm{X}$ - luminescence spectrum, the decay time constant and the light output of these doped samples are presented. Because of the slow decay time these doped lead fluoride samples fall short to be used to build a homogeneous hadronic calorimeter with dual readout for future high energy physics experiments. Researches will continue to introduce scintillation in lead fluoride.
\end{abstract}

\section{INTRODUCTION}

$\mathbf{I}$ NORGANIC crystal scintillators have played an important role in the construction of total absorption electromagnetic calorimeter (ECAL) for high energy physics experiments. Crystals have recently also been proposed to construct a homogeneous calorimeter, including both electromagnetic and hadronic parts [1] for detectors at the international linear collider. This homogeneous hadronic calorimeter (HHCAL) detector concept removes the traditional boundary between the ECAL and the HCAL, so eliminates the effect of dead materials in the middle of the hadronic shower development. It may also takes advantage of the recently implemented dual readout approach to achieve good energy resolution for hadronic jets by measuring both Čerenkov and scintillation light [2]. Because of the un-precedent volume (70 to 100 $\mathrm{m}^{3}$ ) foreseen for such calorimeter [1], the crystal material must be dense (to reduce the volume), UV transparent (to effective collecting the Čerenkov light) and allows a clear discrimination between the Čerenkov and scintillation light.

Cubic lead fluoride $\left(\mathrm{PbF}_{2}\right)$ has a high density of $7.77 \mathrm{~g} / \mathrm{cm}^{3}$ and a short radiation length $\left(\mathrm{X}_{0}=0.93 \mathrm{~cm}\right)$ and a short nuclear interaction length $\left(\lambda_{I}=21 \mathrm{~cm}\right)$. It has good UV transparency down to $250 \mathrm{~nm}$. Its low melting point $\left(824^{\circ} \mathrm{C}\right)$ and low material cost (1/3 of BGO) makes it potentially a cost-effective material for the HHCAL detector concept. Large size $(20 \mathrm{~cm}$ long) $\mathrm{PbF}_{2}$ crystals are available in the market. They were used as a Čerenkov material to construct the ECAL for the A4 experiment at the MAinzer MIcrotron (MAMI) facility in Mainz. Excellent energy resolution was reported [3].

As a Čerenkov material $\mathrm{PbF}_{2}$ has been studied in details [4]. The only issue for the HHCAL application is that it is not a scintillator. Effort was made to introduce scintillation light into $\mathrm{PbF}_{2}$ through phase transition (cubic to orthorhomic) during

This work was supported in part by the U.S. Department of Energy under grant DE-FG03-92-ER-40701 and the U.S. National Science Foundation Award PHY-0612805.

The Authors are with the California Institute of Technology, Pasadena, CA 91125 USA (e-mail: zhu@hep.caltech.edu). growth. Positive result was reported by Klassen et al. [5], but was not confirmed by Derenzo et al. [6]. Observation of fast photo- and $\mathrm{x}$ - luminescence in $\mathrm{Gd}$ doped $\mathrm{PbF}_{2}$ crystals was first reported by Shen et al. of SIC [7], and was later confirmed by Woody et al. in a beam test at AGS [8]. In this work lead fluoride samples doped with various rare earth elements were grown by modified Bridgman method. Photo- and X- luminescence spectrum, decay kinetics and $\gamma$ ray excited anode current and pulse height spectrum were measured.

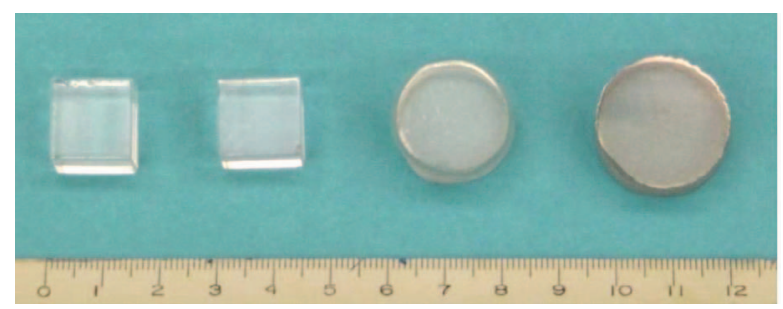

Fig. 1. A photo showing lead fluoride crystal samples doped with various rare earth elements from SIC (left two cubes) and Scintibow (right two cylinders).

A total of $116 \mathrm{PbF}_{2}$ samples doped with various rare earth elements were grown by modified Bridgman method at Shanghai Institute of Ceramics (SIC) and Shanghai Scintibow Crystal Co., LTD. While classical platinum crucible was used at SIC for the crystal growth, graphite crucible was used at Scintibow. The SIC samples are cubes of 1.5 radiation length with six faces polished as shown in the left two samples in Fig.1. Most Scintibow samples are cylinders with two end faces polished and have a dimension of $\phi 22 \times 15 \mathrm{~mm}$ as shown in the right two samples in Fig.1. Crystals grown in graphite crucible are less transparent that in platinum crucible.

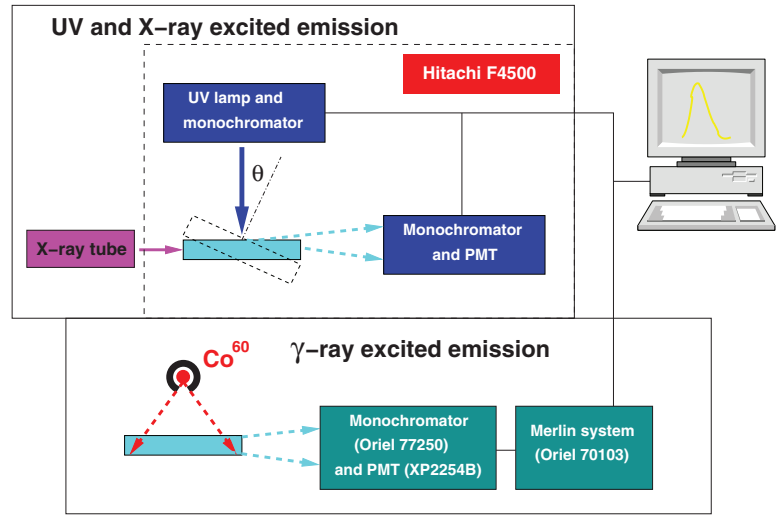

Fig. 2. The setup used for the photo- and $\mathrm{x}$ - luminescence measurement. 


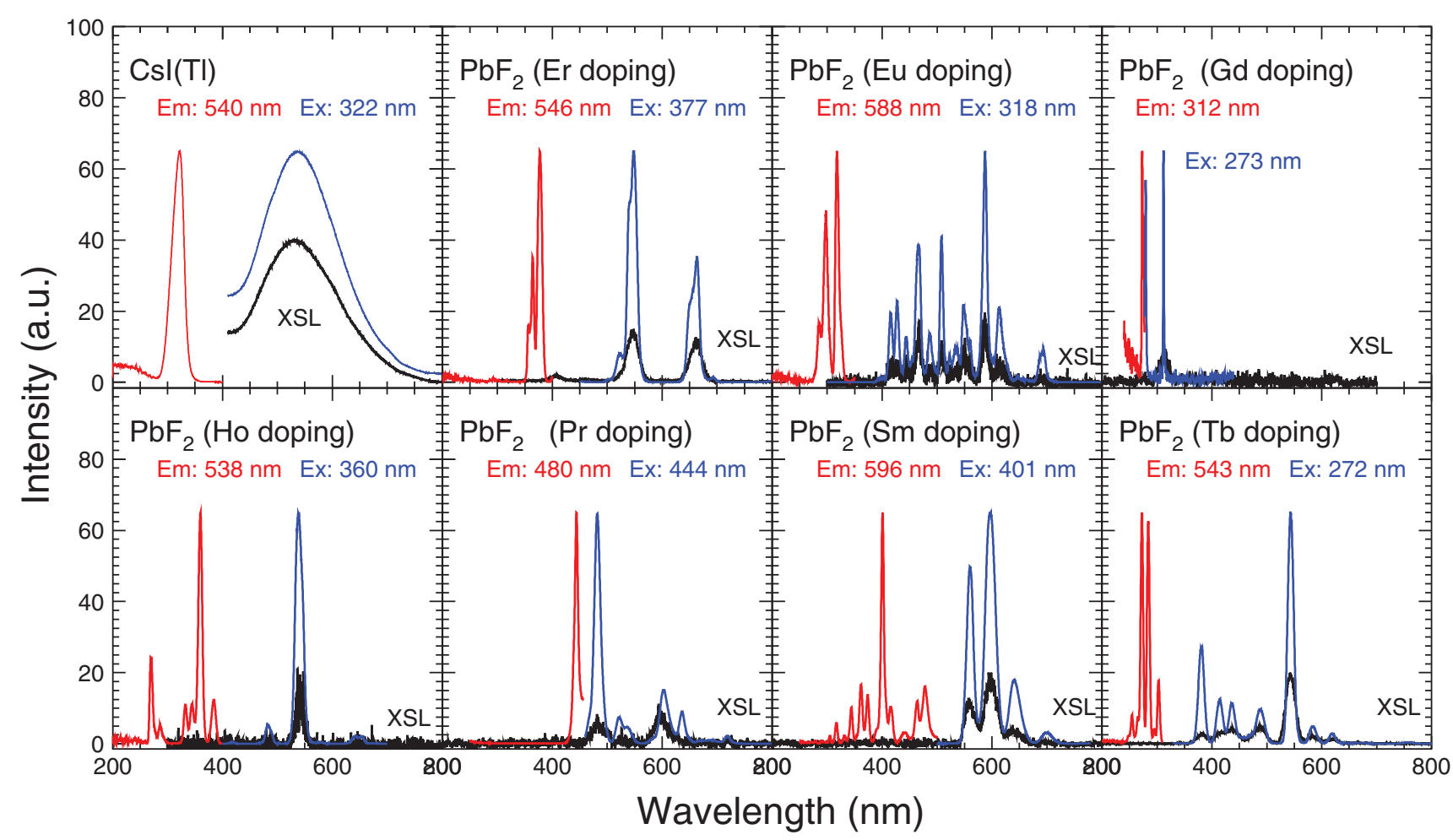

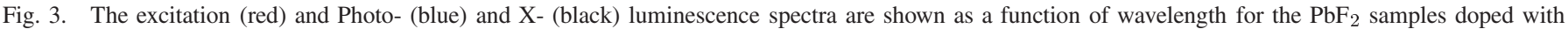
$\mathrm{Er}, \mathrm{Eu}, \mathrm{Gd}, \mathrm{Ho}, \mathrm{Pr}, \mathrm{Sm}$ and $\mathrm{Tb}$ as well as a reference $\mathrm{CsI}(\mathrm{Tl})$ sample.

\section{Photo- And X- Luminescence Spectrum}

Photo- and X-ray luminescence spectrum was measured by a Hitachi F-4500 fluorescence spectrophotometer. Fig. 2 shows the set-up used for this measurement. For the X-ray luminescence measurement an AMTPEK E3-T X-ray tube was run at $25 \mathrm{kV}$ and $50 \mu \mathrm{A}$. Fig. 3 shows excitation (red) photo-luminescence (blue) and X-luminescence (black) spectra for the $\mathrm{PbF}_{2}$ samples doped with $\mathrm{Er}, \mathrm{Eu}, \mathrm{Gd}, \mathrm{Ho}, \mathrm{Pr}, \mathrm{Sm}$ and $\mathrm{Tb}$ as well as a reference $\mathrm{CsI}(\mathrm{Tl})$ sample. Although spectroscopic resolutions are different, the observed photoand $\mathrm{X}$ - luminescence spectra are consistent for all samples.

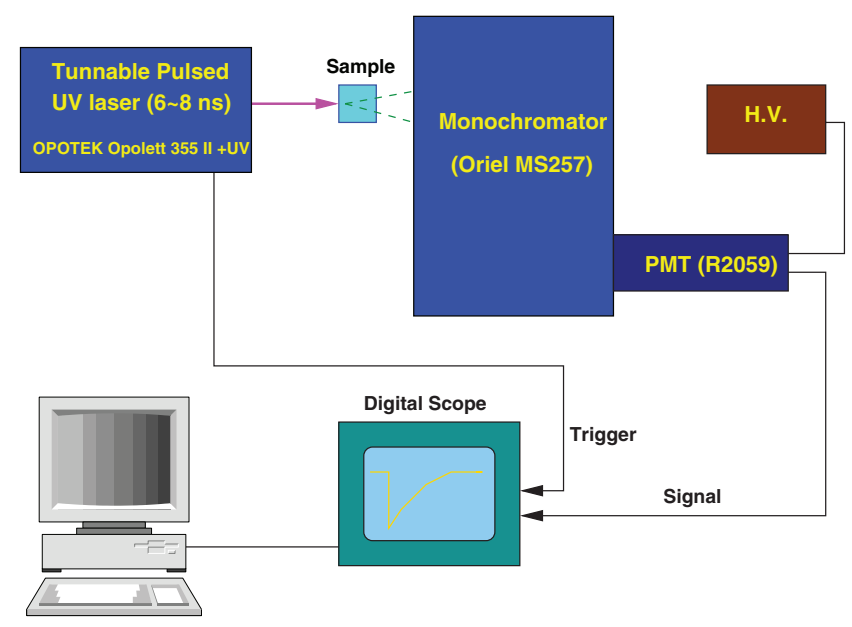

Fig. 4. The setup used for the photo-luminescence pulse shape and the decay time measurement.
The photo- and $\mathrm{x}$ - luminescence spectra observed in the $\mathrm{Gd}$ doped $\mathrm{PbF}_{2}$ samples are consistent with previous publications by Shen and Woody [7], [8].

\section{Photo-luminescence Decay Time Constant}

The photo-luminescence decay time constant of these doped $\mathrm{PbF}_{2}$ samples was measured by using a pulsed laser as the excitation source. Fig. 4 shows the set-up used in this measurement. The UV light pulses of $6-8 \mathrm{~ns}$ width from an OPOTEK Opolette $355 I I+U V$ tunable laser was used as the excitation source. The photo-luminescence light from these samples went first through an Oriel MS257 monochromator then to a Hamamatsu R2059 PMT. The luminescence pulse shape observed by the PMT was recorded by an Agilent 6052A digital scope. The decay time constants were determined by an exponential fit to the pulse shape. Fig. 5 shows the photoluminescence pulse shape (blue), the corresponding exponential fit (red dots) and decay time constant for the $\mathrm{PbF}_{2}$ samples doped with $\mathrm{Er}, \mathrm{Ho}, \mathrm{Eu}, \mathrm{Sm}$ and $\mathrm{Tb}$ as well as a reference CsI(Tl) sample. Table I summarizes the decay time constants for samples doped with rare earth, which were found at a millisecond scale. This time constant is too long to be useful for high energy physics experiments.

TABLE I

DeCay Time Constant For Doped Lead Fluoride CRystals

\begin{tabular}{cccccc}
\hline \hline Dopant & $\mathrm{Er}$ & $\mathrm{Eu}$ & $\mathrm{Ho}$ & $\mathrm{Sm}$ & $\mathrm{Tb}$ \\
\hline Decay time constant $(\mathrm{ms})$ & 1.5 & 8.5 & 1.3 & 7.0 & 5.0 \\
\hline \hline
\end{tabular}



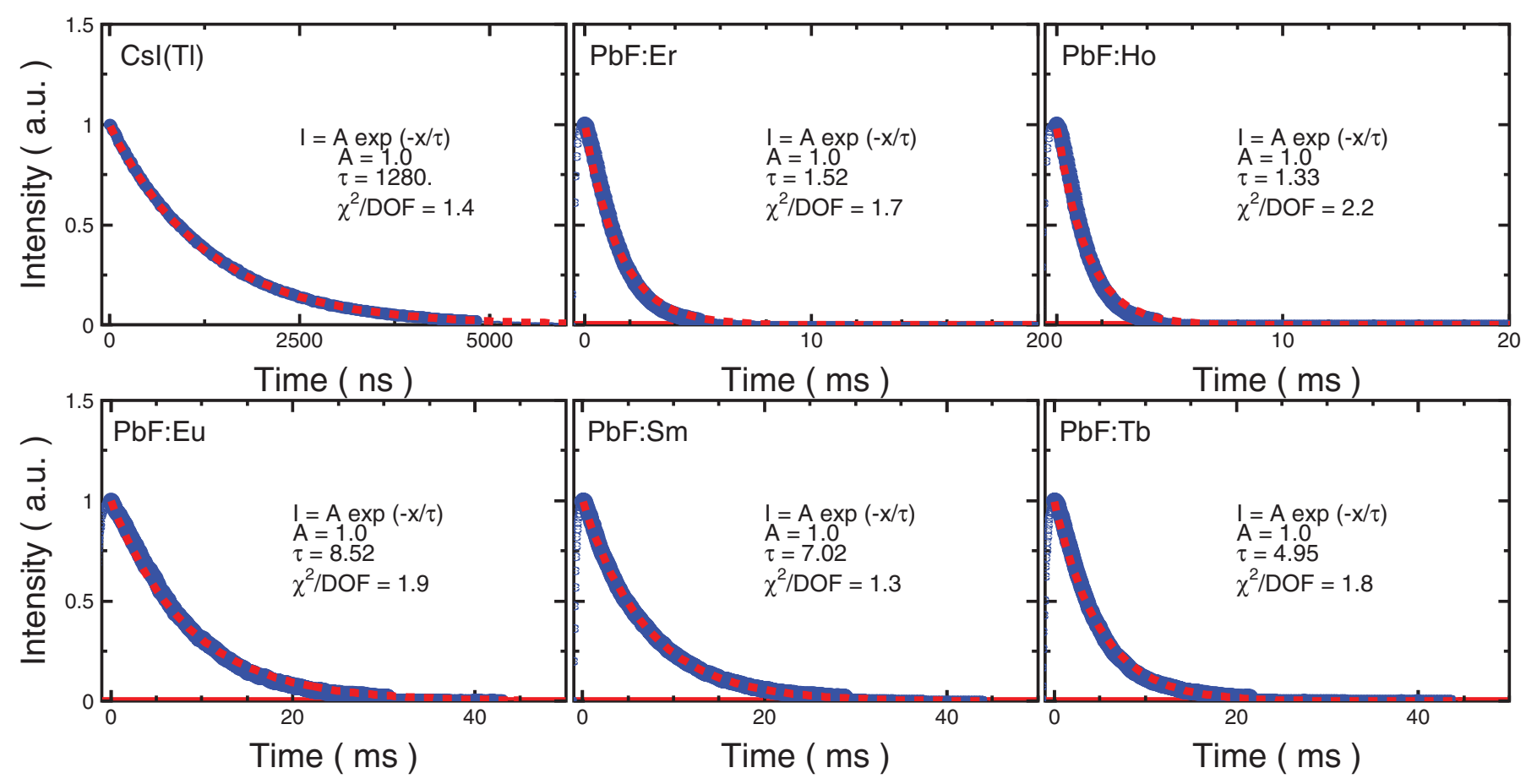

Fig. 5. The photo-luminescence pulse shape (blue), corresponding fit to an exponential (red dots) and the decay time constant are shown for the PbF 2 samples doped with $\mathrm{Er}, \mathrm{Ho}, \mathrm{Eu}, \mathrm{Sm}$ and $\mathrm{Tb}$ as well as a reference CsI(Tl) sample.

\section{IV. $\gamma$-RAY INDUCED ANODE PHOTO-CURRENT}

Fig. 6 shows the setup used for the anode current measurement. A ${ }^{137} \mathrm{Cs}$ source was used to excite the samples. A Hamamatsu R2059 PMT was used to measure the anode photo-current. The bias voltage of the PMT was fixed at $2,000 \mathrm{~V}$, and the distance between the source and the samples was fixed at $2 \mathrm{~cm}$. The PMT anode current was measured by using a digital multi-meter.

Fig. 7 and Fig. 8 show the anode photo-current measured respectively for all $\mathrm{PbF}_{2}$ samples (black and blue dots) and a

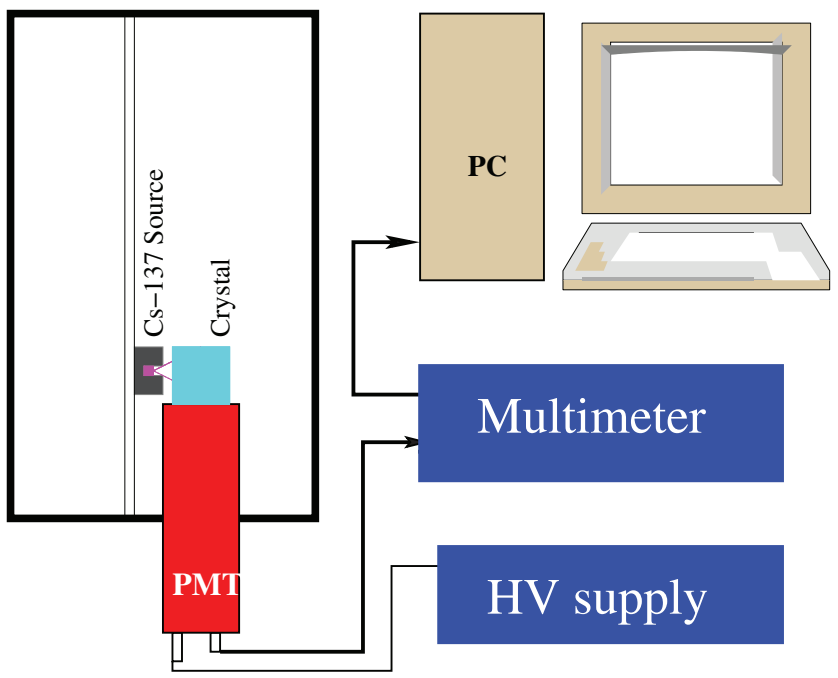

Fig. 6. The setup used to measure the $\gamma$-ray induced anode photo-current for $\mathrm{PbF}_{2}$ samples. The distance between source and samples was fixed at 2 $\mathrm{cm}$. un-doped $\mathrm{PbF}_{2}$ sample (red). The corresponding anode photocurrent of a reference PWO sample with light output of 20 p.e./MeV is also shown in Fig. 8. The anode photo-current was found to be $42 \mathrm{nA}$ and $240 \mathrm{nA}$ respectively for the undoped $\mathrm{PbF}_{2}$ and the PWO sample. A total of six doped $\mathrm{PbF}_{2}$ samples showed an anode photo-current of larger than 50

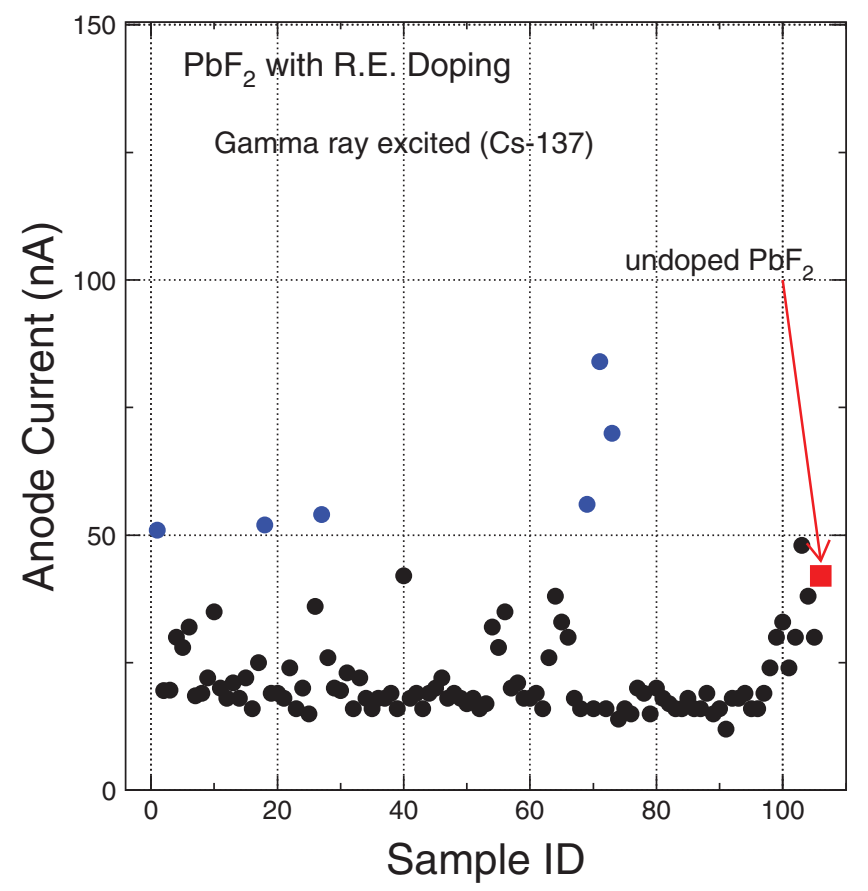

Fig. 7. The PMT anode photo-current measured for all $\mathrm{PbF}_{2}$ samples (black and blue dots). 


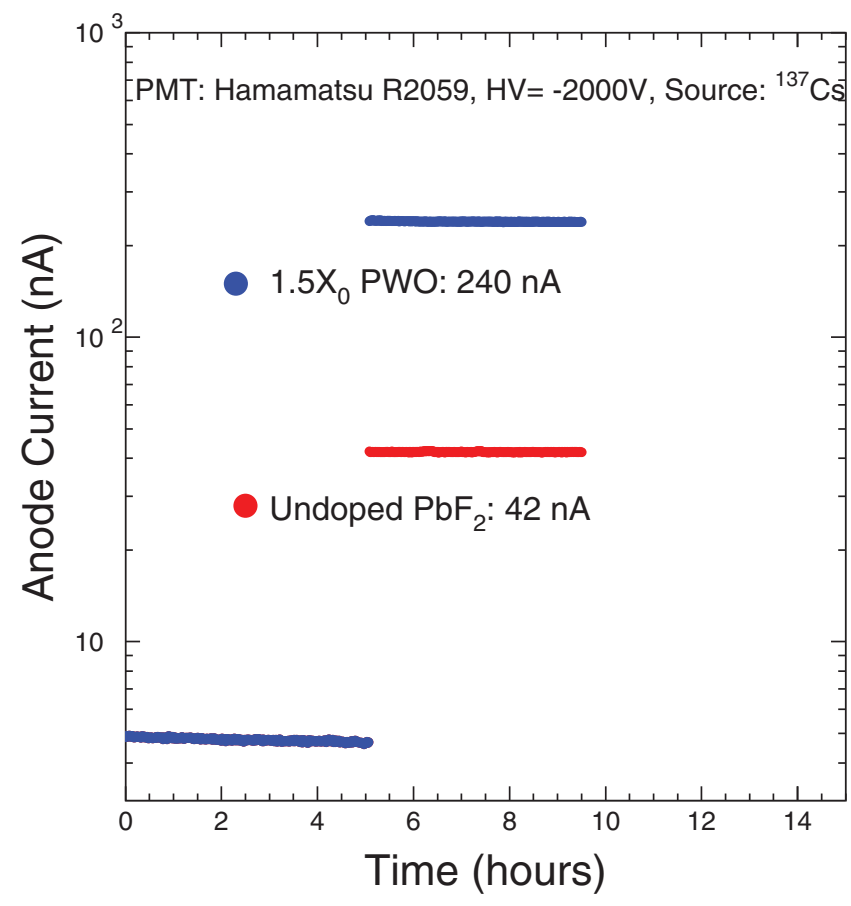

Fig. 8. The PMT anode current measured for a un-doped $\mathrm{PbF}_{2}$ sample (red) and a reference small PWO sample (blue) with light output of 20 p.e. $/ \mathrm{MeV}$

nA, which are marked as the blue dots in Fig. 7. Table II lists their ID, dimension and dopant. The numerical result of this measurement indicates that the light output of these doped $\mathrm{PbF}_{2}$ samples are less than 3 p.e./MeV. We also note that all these samples are doped with $\mathrm{Eu}$, and were grown at Scintibow.

TABLE II

The PMT Anode CURRENT For Doped PBF 2 SAMPles

\begin{tabular}{cccc}
\hline \hline ID & Anode Current (nA) & Size $(\mathrm{mm})$ & Doping \\
\hline Scintibow-1 & 51 & $18 \times 12 \times 10$ & $\mathrm{Eu}$ \\
Scintibow-18 & 52 & $\phi 22 \times 15$ & $\mathrm{Eu} / \mathrm{Gd}$ \\
Scintibow-27 & 53 & $\phi 20 \times 15$ & $\mathrm{Eu} / \mathrm{Tb}$ \\
Scintibow-B19 & 56 & $\phi 20 \times 15$ & $\mathrm{Eu} / \mathrm{Tb} / \mathrm{Na}$ \\
Scintibow-B21 & 83 & $\phi 22 \times 15$ & $\mathrm{Eu} / \mathrm{Bi} / \mathrm{Na}$ \\
Scintibow-B23 & 73 & $\phi 20 \times 15$ & $\mathrm{Eu} / \mathrm{Bi} / \mathrm{Na}$ \\
\hline \hline
\end{tabular}

\section{V. ${ }^{137} \mathrm{Cs} \gamma$-RAy Exited Pulse Height Spectrum}

${ }^{137} \mathrm{Cs} \gamma$-ray exited pulse height spectrum was measured for doped $\mathrm{PbF}_{2}$ samples listed in table II. A Hamamatsu R2059 PMT was used as the readout device with an integration gate of $1 \mu \mathrm{s}$. Fig. 9 and Fig. 10 show the pulse height spectra for samples Scintibow-1 and Scintibow-B21 respectively. Also shown in these figures is the pulse height spectrum for a undoped $\mathrm{PbF}_{2}$ sample. There is very little difference between spectra obtained from the doped and the un-doped $\mathrm{PbF}_{2}$ samples, indicating that the amount of the luminescence light in the $1 \mu$ s gate is too weak to show a peak.

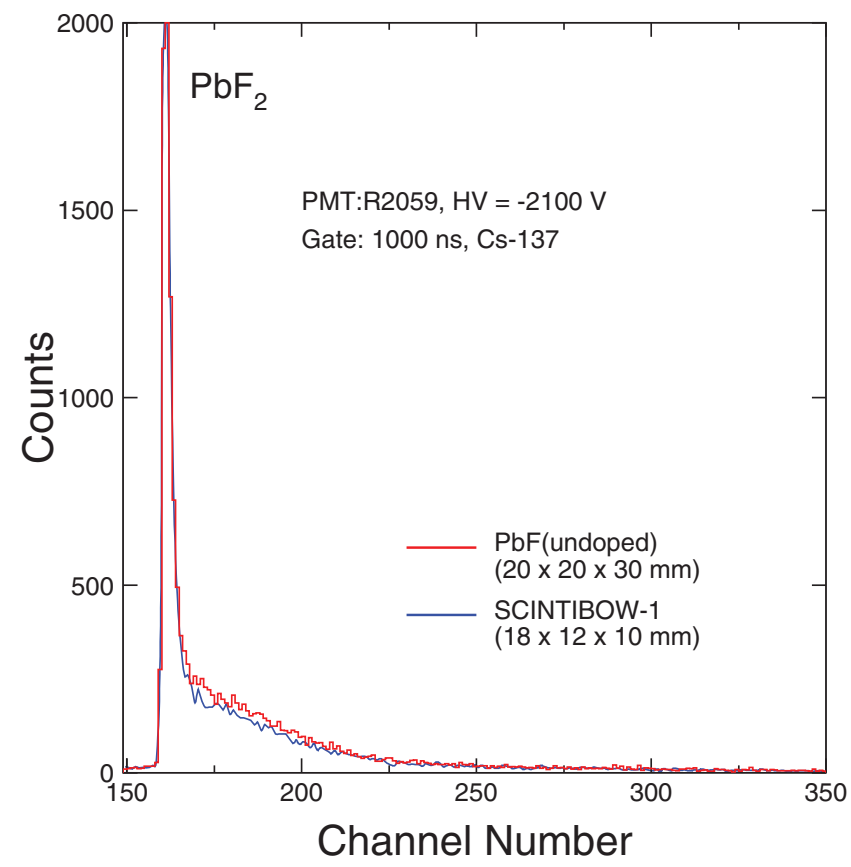

Fig. 9. ${ }^{137} \mathrm{Cs} \gamma$-ray exited pulse height spectrum for doped $\mathrm{PbF}_{2}$ samples Scintibow-1.

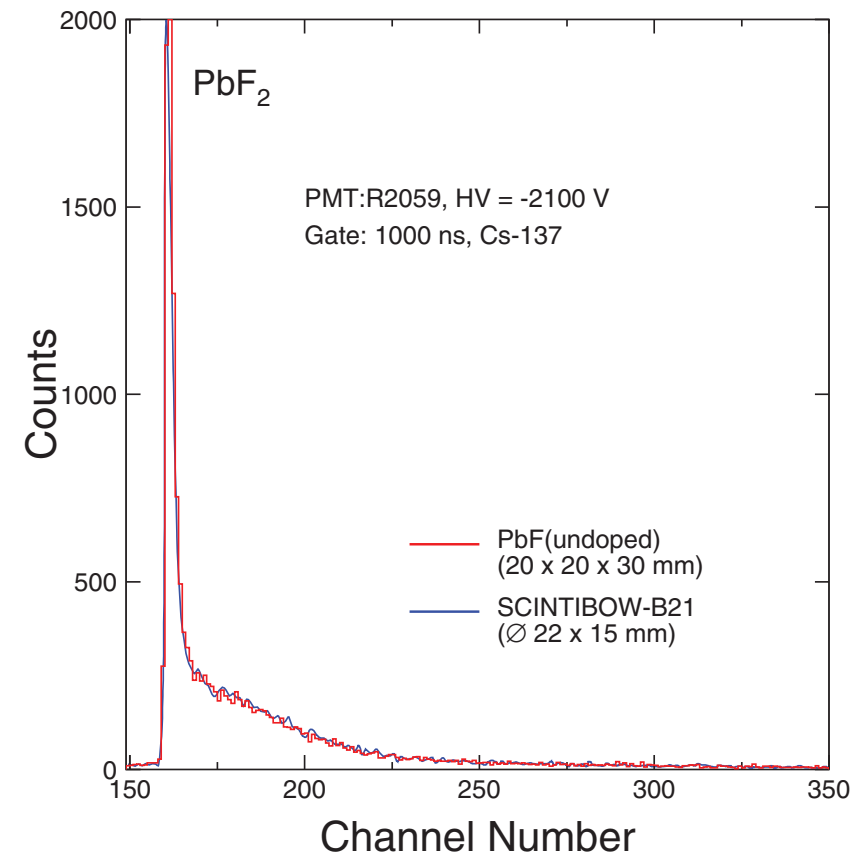

Fig. 10. ${ }^{137} \mathrm{Cs} \gamma$-ray exited pulse height spectrum for doped $\mathrm{PbF}_{2}$ samples Scintibow-B21.

\section{SUMMARY}

A search for scintillation was carried out in a set of doped lead fluoride crystal samples. Consistent photo- and X-ray luminescence spectra were found in $\mathrm{PbF}_{2}$ samples doped with Er, Eu, Gd, Ho, Pr, Sm and Tb. The decay time of these doped samples was found to be at a millisecond scale as expected from the $\mathrm{f}-\mathrm{f}$ transition of these rare earth elements. While some doped samples show $\gamma$-ray induced anode photo-current larger then the un-doped sample the numerical result indicates that the scintillation light, if any, is less than 3 p.e./MeV measured 
by a PMT with bi-alkali photo-cathode. Their ${ }^{137} \mathrm{Cs} \gamma$-ray excited pulse height spectra measured with $1 \mu$ s integration gate were found identical to un-doped sample, confirming that their luminescence in the $1 \mu$ s gate is too weak to show a peak. Investigation will continue to search for scintillation in doped lead fluoride for the HHCAL detector concept.

\section{ACKNOWLEDGMENTS}

The authors would like to thank Prof. Dingzhong Shen of Scintibow and Prof. Guohao Ren of SIC for providing doped $\mathrm{PbF}_{2}$ samples used in this study.

\section{REFERENCES}

[1] A. Para, talk given in International Linear Collider Workshop 2008 at Chicago.

[2] R. Wigmans, in Proceedings of VII International Conference on Calorimetry in Particle Physics, Ed. E. Chen et al., World Scientific (1998) 182, and N. Akchurin et al., Nucl. Instr. and Meth. A537 (2005) 537.

[3] F. Maas, in Proceedings of Xth Pisa Meeting on Advanced Detectors (2006).

[4] D. Anderson and C. Woody et al., Nucl. Instr. and Meth. A290 385 (1990) and IEEE Trans. Nucl.Sci. NS-40 546 (1993).

[5] N. Klassen et al., Heavy Scintillators, ed. F. De Notaristefani et al., Editions Frontieres, 587 (1992).

[6] S. Derenzo et al., IEEE Trans. Nucl.Sci. NS-37 206 (1990) and Nucl. Instr. and Meth. A342 473 (1994).

[7] D. Shen et al., Jour. Inor. Mater. Vol 101 (1995) 11.

[8] C. Woody et al., IEEE Trans. Nucl. Sci. 43 (1996) 1303. 